\title{
Mobile Robot Moving Target Detection and Tracking System
}

Shujing Zhang ${ }^{1}$

Electricity and Information Engineering, Beijing University of Civil Engineering and Architecture

Beijing, 100044, China

E-mail: $152011092850163 . \mathrm{com}$

\section{Lei Zhang ${ }^{2}$}

Electricity and Information Engineering, Beijing University of Civil Engineering and Architecture Beijing, 100044, China

E-mail: zhangleidbucea.edu.cn

\section{Ri Gao}

Electricity and Information Engineering, Beijing University of Civil Engineering and Architecture Beijing, 100044, China

E-mail: $136914167070163 . \mathrm{com}$

\section{Chenxi Liu}

Electricity and Information Engineering, Beijing University of Civil Engineering and Architecture

Beijing, 100044, China

E-mail: 1102286596 @qq. com

In order to solve the problem that the robot can coexist harmoniously with human beings and provide better service in the human settlement environment. Based on the Mecanum wheel mobile robot platform, by introducing the pyramid optical flow algorithm and CamShift algorithm, empirical results show that the robot can quickly predict the movement tendency and speed of the moving object according to the moving characteristics and color characteristic information of the moving object as well as accurate identification of tracking. Based on the above algorithm research, it can effectively improve the ability of robot motion planning and executing tasks in complex environment.

CENet2017

22-23 July, 2017

Shanghai, China

${ }^{1}$ Correspongding Author

${ }^{2}$ This study is supported by NSFC under Grant NO.: 61473027 and Beijing Key Laboratory for Biomimetic and Function of Robot Grant BZ0337. 


\section{Introduction}

The degree of intelligence of the robot mainly depends on its perception of the surrounding environment. In this paper, the software development environment of mobile robot is VS2010 and OpenCV2.4.9. The robot acquires two-dimensional image of the environment through the visual acquisition system and extracts the object image feature by the algorithm processing, converting it to the image information that the robot can recognize and judge the movement of the target object based on the image information. In this paper, the novel visionbased detection system is used to detect and track the moving targets from near-to-far ranges and the tracking process is applied after detectionI. It is used to track and predict the future motion and direction of pedestrian [1]. Speed Up Robust Feature (SURF) algorithm is used for detection, continuous tracking and recognition. This paper uses the SURF on the moving human target based on the shirts pattern [2]. To efficiently associate the detected human frame by frame, they propose a novel human tracking framework in combination with the constrainedmultiple-kernel tracking and the estimated 3-D information (depth) to globally optimize the data association between consecutive frames [3].

\section{Mobile Robot Platform}

Mecanum wheel four-wheel mobile robot can realize forward, backward, left and right movement, 45 degrees oblique movement, 360 degrees all round zero radius and other actions. In this paper, the wheel diameter of $15 \mathrm{~cm}$ and thickness of $3.5 \mathrm{~cm}$, there are 16 of the 45 degrees driven roller. The robot platform size is $60 \mathrm{~cm} \times 45 \mathrm{~cm}$. Using DC motor drive, current and speed dual closed-loop control method. The robot drives the battery with a $24 \mathrm{~V} 10 \mathrm{AH}$ DC battery.

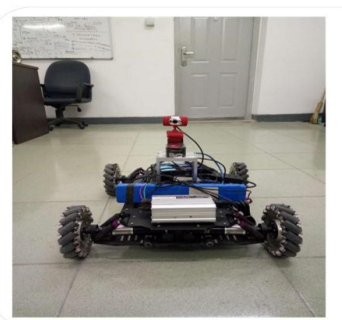

Figure1: Robot Prototype Figure 2: Multi-mode Movement

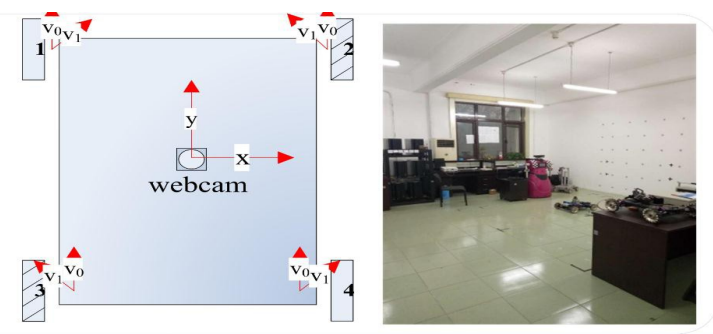

Figure 3: The Environment

\section{Visual System Objective Task Decomposition}

\subsection{Image Preprocessing}

The image acquisition and preprocessing, feature analysis, color moving target detection and tracking, are mainly to raise the quality of the image so as to reduce the error rate in the process of target detection. The visual system workflow is shown as follows:

\begin{tabular}{|c|c|c|c|c|}
\hline Moving target & $\begin{array}{l}\text { Video image } \\
\text { acquisition }\end{array}$ & $\begin{array}{c}\text { Image } \\
\text { preprocessing }\end{array}$ & $\begin{array}{c}\text { Target } \\
\text { Recognition }\end{array}$ & Target Tracking \\
\hline
\end{tabular}

The color image is processed with gray scale and the channels are weighted average: Gray $=0.299 \mathrm{R}+0.587 \mathrm{G}+0.114 \mathrm{~B}$. The Canny edge detection algorithm is used to calculate the gradient amplitude and direction: 


$$
G=\sqrt{G_{x}^{2}+G_{y}^{2}}, \theta=\arctan \left(\frac{G_{y}}{G_{x}}\right)
$$

\subsection{Pyramid Lucas-Kanade Optical Flow Target Detection Algorithm}

The optical flow method is the instantaneous velocity of the pixel motion of the space moving object on the observed imaging plane. When the object is moving, it is also in the image corresponding to the brightness mode, so efforts have to be made to help the robot determine the movement of the target. If there is a relative motion at the background of the image, the velocity vector formed by the moving object is different from the velocity vector of the neighborhood background [3].

Assuming that $\mathrm{I}$ and $\mathrm{J}$ are two grayscale images, the gray value for each pixel on the image is defined as: $I(x)=i(x, y), J(x)=j(x, y)$

Where $x=(x, y)$ is the image coordinates of the pixel on the image. For the pyramid tracking purposes, the previous frame of Image I on a point $u(u x, u y), d=\left[d_{x}, d_{y}\right]$, in the latter frame of Image $\mathbf{J}$ to find a point $v(u x+d x, u y+d y)$ to match, the transformation matrix is defined as:

$$
A=\left[\begin{array}{cc}
1+d_{x x} & d_{x y} \\
d_{y x} & 1+d_{y y}
\end{array}\right]
$$

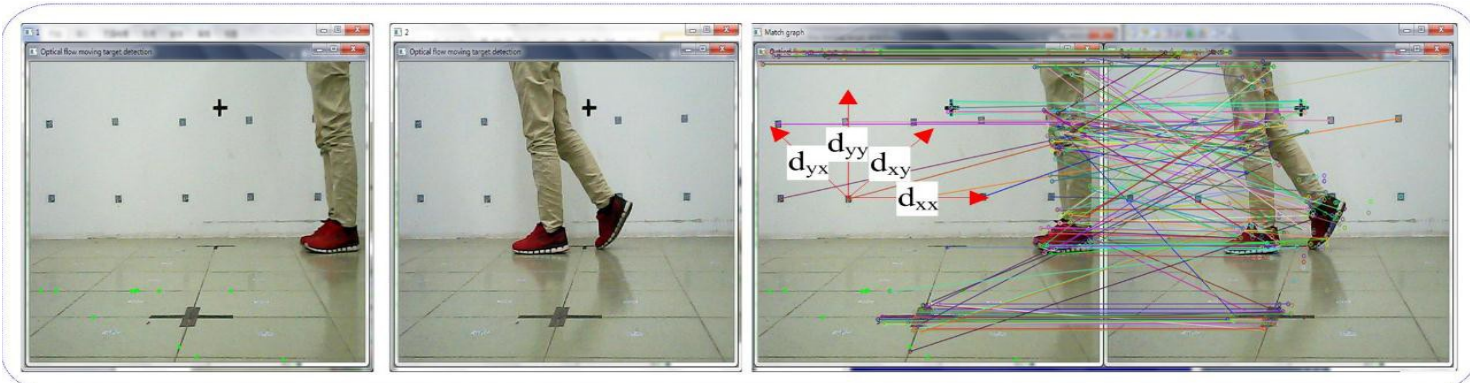

Figure 4: Affine Deformation Graph

Where four parameters ${ }^{d_{x x}},{ }^{d_{y y}}, d_{x y}$ and ${ }^{d_{y x}}$ represent the affine deformation in the image.

The error as: $\varepsilon(d, A)=\varepsilon\left(d_{x}, d_{y}, d_{x x}, d_{x y}, d_{y X}, d_{y y}\right)=\sum_{x=-W_{x}}^{W_{x}} \sum_{y=-W_{y}}^{W_{y}}(I(x+u)-J(A x+d+u))^{2}$

This paper improves the small and coherent movements, the image of pyramid thought into the optical flow method and multi-resolution to represent the image. When the image is 400 $\times 400$, the object velocity is $[16,16]$, then the image is reduced to $200 \times 200$, the speed becomes $[8,8]$, reduced to $100 \times 100$ and the speed reduced to $[4,4]$. Therefore, for the original image after multiple zoom, the original algorithm is applicable [4]. 


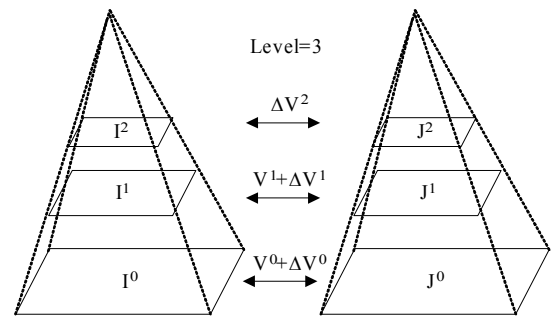

Figure 5: Pyramid Optical Flow Model

\subsection{CamShift Moving Target Tracking Algorithm}

The Camshift tracking algorithm is a target tracking method with color histogram as the target model. Camshift algorithm firstly calculates the color probability distribution of the video image, then initializes the search window in the first frame of the video image, automatically adjusts the position and size of the search window, and realizes the tracking of the target. In the next frame video image, with the previous frame in the target object location and size as the initial search window, determine the target location and size of the new [5]. In the search window of the center of the window, when the zero-order moves $M_{00}$, the first-order moment $M_{10}, M_{01}$ and the second-order moment $M_{20}, M_{02}, M_{11}$ :

$$
\begin{aligned}
M_{00}=\sum_{x} \sum_{y} I(x, y) M_{10} & =\sum_{x} \sum_{y} x I(x, y) \quad M_{01}=\sum_{x} \sum_{y} y I(x, y) \\
M_{20}=\sum_{x} \sum_{y} x^{2} I(x, y) M_{02} & =\sum_{x} \sum_{y} y^{2} I(x, y), M_{11}=\sum_{x} \sum_{y} x y I(x, y)
\end{aligned}
$$

The center position of the search window: $x_{c}=M_{10} / M_{00}, y_{c}=M_{01} / M_{00}$, the calculation result as the center of the search window in the next frame. Adjust the size of the search window according to $M_{00} .1$ and w are the length and width of the search window. The direction angle of the long axis of the ellipse tracking frame of the moving object is:

$$
\begin{gathered}
I=\sqrt{\frac{(a+c)+\sqrt{b^{2}+(a-c)^{2}}}{2}}, W=\sqrt{\frac{(a+c)-\sqrt{b^{2}+(a-c)^{2}}}{2}} \\
a=\frac{M_{20}}{M_{11}}-x_{c}^{2}, b=2\left(\frac{M_{11}}{M_{00}}-x_{c} y_{c}\right), c=\frac{M_{02}}{M_{11}}-y_{c}^{2}, \theta=\frac{1}{2} \tan ^{-1}\left(\frac{b}{a-c}\right)
\end{gathered}
$$

The zero-order moment is the sum of all pixels in the search window. After reverse projection of the target $\mathrm{H}$ component histogram, most of the normalized value of the target area of the search window should be the maximum value of 255 ; if the calculated zero order moment is greater than a certain threshold, it can be considered at this time the target coveres the entire search window. There is reason to believe that there is still a target area outside the search window and it needs to increase the size of the search window; accordingly, if the zero moment is less than a certain threshold, you need to narrow the size of the search window. When the size changes, the CamShift algorithm can be adapted to adjust the target area to track. In this paper, use the region of interest (ROI) in Image [6]. The algorithm's specific implementation process is shown as follows: 


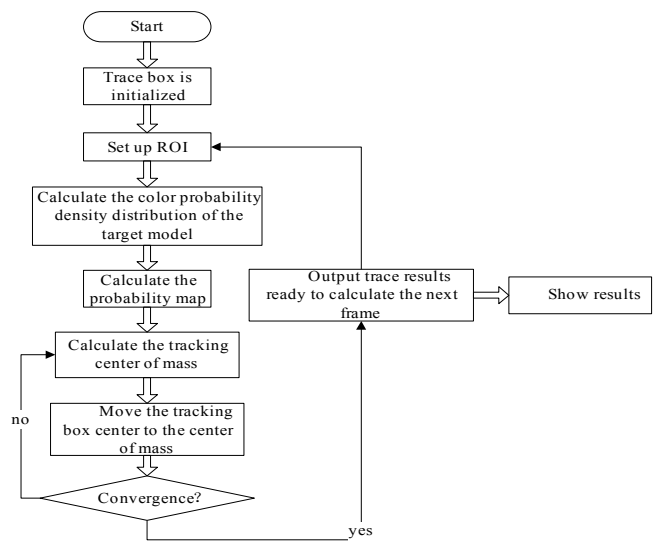

Figure 6: Camshift Tracking Algorithm Flow Chart

\section{Experimental Results}

In the experiment, the robot controller uses the industrial microcomputer $(2.16 \mathrm{GHz}$ processor, 4.00GB RAM) as the hardware platform, vs2010 and OpenCV2.4.9 for the software operating environment. In the laboratory environment, the size of the image is $320 \times 240$ and the image sequence includes a moving body [7].

\subsection{Pyramid Optical Flow Target Detection Algorithm Experimental}

In Experiment 1, the use of OpenCV in the VideoCapture class call the camera and the code VideoCapture capture and capture.open (1) to achieve video capture. In the experiment, the robot can determine the direction and size of the moving target.

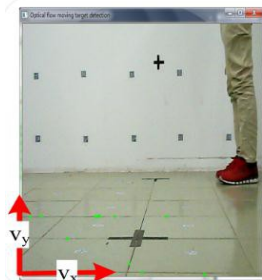

Figure 7: 98 frame

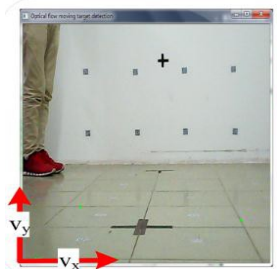

Figure 8: 270 frame

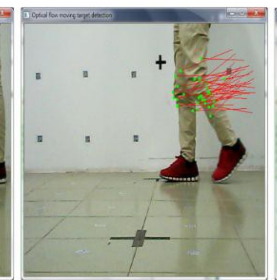

113 frame

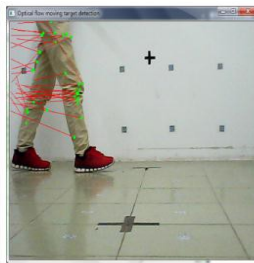

292 frame

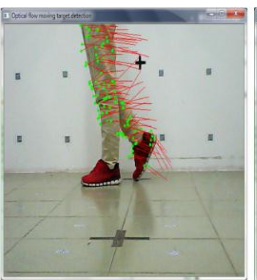

127 frame

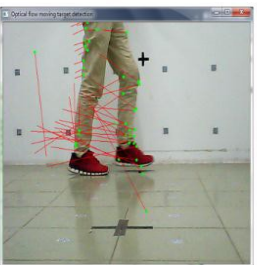

307 frame

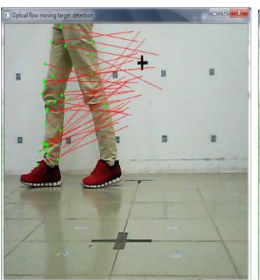

134 frame

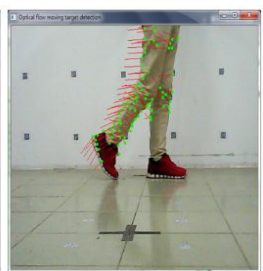

313 frame

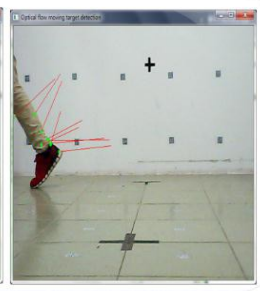

159 frame

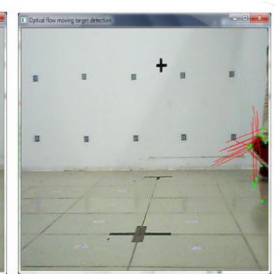

327 frame

From the above experiments, we can see that from the 98 frame to the 270 frame turn back again, the algorithm can detect the movement trends and size of the object in a more quickly and accurately manner. The background of the optical flow is 0 and select a smaller threshold when the optical flow is greater than the threshold to determine the moving target. The pyramid optical flow method can calculate the corresponding motion vector for each pixel. When the moving body moves at normal pace, the green dots indicate the trend of the moving direction of the object. The velocity of the moving object can be determined by the length of the red line.

Among them, the motion vector $\mathrm{X}$-axis and $\mathrm{y}$-axis component is value $\overline{v_{X}}$ and value $\overline{v_{y}}$ [8]. 


\subsection{CamShift Moving Target Tracking Algorithm}

In Experiment 2, the robot through CamShift tracking algorithm, can effectively track the moving target within the field of vision by using the moving target color histogram.

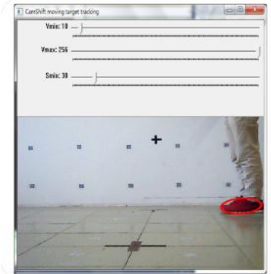

Figure 9: 98 frame

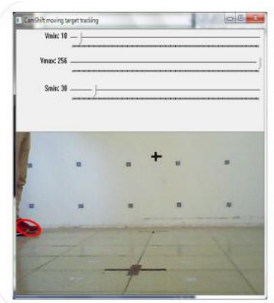

Figure 10:210 frame

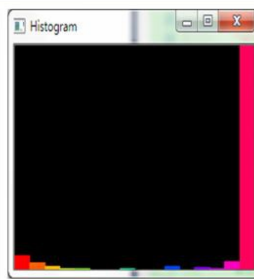

Histogram

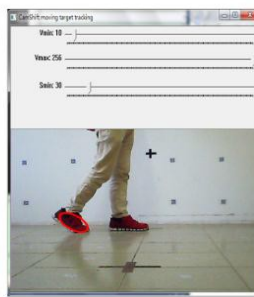

238 frame

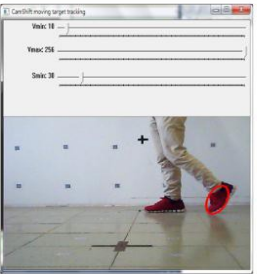

102 frame

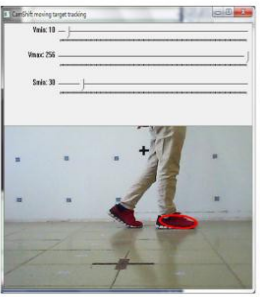

250 frame

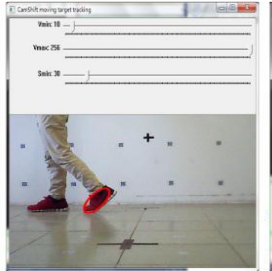

159 frame

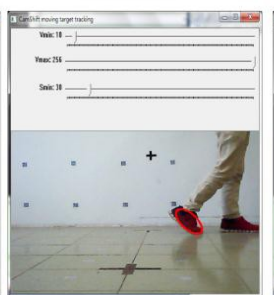

272 frame

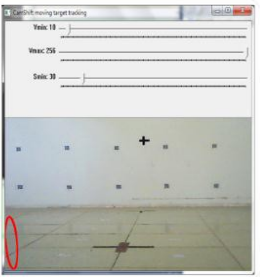

168 frame

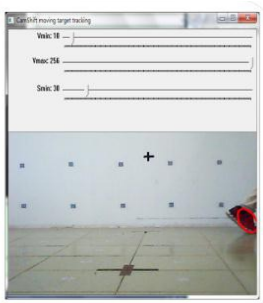

286 frame

The CamShift algorithm establishes a color histogram for the $\mathrm{H}$ component in the HSV color model as the color information mainly concentrates in the $\mathrm{H}$ component, which can effectively reduce the light changes to the tracking interference. Firstly, the box is selected to track the moving body oval search box, get the moving target color histogram, access the current moving target location and area size [9]. As seen from the figure, in the 98th frame to select the moving target, the formation of histogram as shown in the left foot color histogram until the 159th track tracking effect is better. When the 168th moving target is out of the robot vision, the search box is still valid and there is no target loss situation, starting from the 210th, the movement of the human body turned again in the robot field of vision, the right foot as a moving target; so the algorithm can be a good detection of the shoes inside and outside the color of the difference, until out of sight; it is also tracking the target.

In addition, when the target object appears in case of speed mutation, overlap and other irregular motion state, which will be shown in the Figure 11:
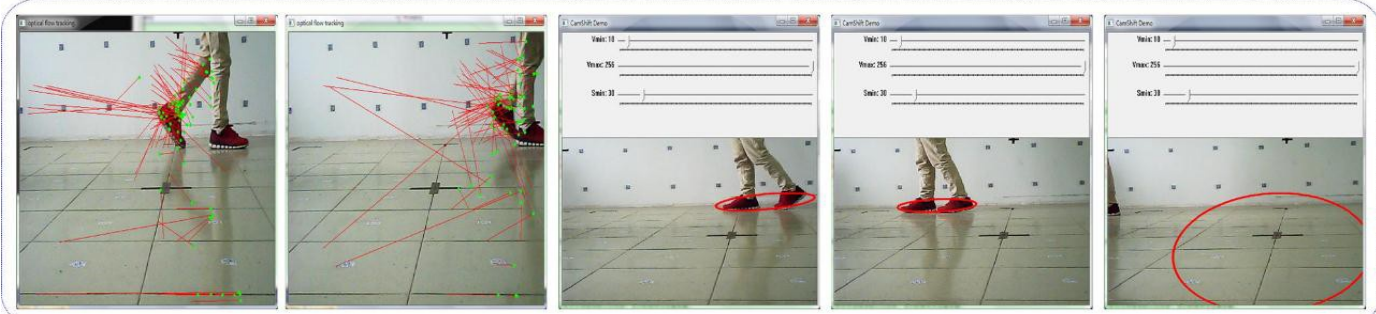

Figure 11: Speed Mutation, Overlap and Other Rrregular Motion State

1) Making the pyramid optical flow algorithm to generate part of the interference motion detection, resulting in divergent shortcomings; 2)the tracking algorithm search box to expand the search target, resulting in similar color moving objects overlap; 3 ) the movement is too fast and the target tracking is lost. 


\section{Conclusion}

In order to verify the feasibility of the above algorithm, this experiment has carried out 80 target detecting and tracking tests, containing a variety of sports state. The statistics are as follows:

\begin{tabular}{|c|c|c|}
\hline Experiment times & Effective detection times & Effective tracking times \\
\hline 80 & 75 & 73 \\
\hline Success rate & $93.75 \%$ & $91.25 \%$ \\
\hline
\end{tabular}

Table 1: Moving Target Detection and Statistics of Tracking Success Rate

In combination with the above experimental results, the pyramid optical flow method and Camshift tracking algorithm can better realize the robot detection of moving pedestrians in the static background according to the direction of the initiative to track the moving target object. This experiment is limited to two-dimensional tracking target. The speed is limited to uniform movement and the tracking accuracy is to be further improved; when the robot vision in the object color and ambient color are similar, it will appear to identify errors, resulting in robot movement and expectations bias or even error. This experiment needs to further strengthen the stability and optimize the algorithm.

\section{References}

[1] A. Mammeri, T. Y. Zuo, A. Boukerche. Extending the Detection Range of Vision-Based Vehicular Instrumentation[J]. IEEE Transactions on Instrumentation and Measurement. 65(4): 856-873(2016)

[2] G. C. How, E. S. L. Ming, Y. C. Fai, T. S. Ho. Shirt pattern recognizing and tracking behavior analysis using SURF on the moving human target[C]. 2015 10th Asian Control Conference (ASCC). IEEE, Kota Kinabalu, Malaysia. 1-5(2015)

[3] K. H. Lee, J. N. Hwang, G. Okopal, J. Pitton. Ground-Moving-Platform-Based Human Tracking Using Visual SLAM and Constrained Multiple Kernels[J]. IEEE Transactions on Intelligent Transportation Systems. 17(12): 3602-3612(2016)

[4] W. Q.Ye, Z. J. Li, C. G.Yang, J. J. Sun, C. Y. Su, R. Q. Lu. Vision-Based Human Tracking Control of a Wheeled Inverted Pendulum Robot[J]. IEEE Transactions on Cybernetics. 46(11): 24232434(2016)

[5] M. Gupta, L. Behera, V. K. Subramanian, M. M. Jamshidi. A Robust Visual Human Detection Approach With UKF-Based Motion Tracking for a Mobile Robot[J]. IEEE Systems Journal. 9(4): $1363-1375(2015)$

[6] H. Zhang, C. Reardon, L. E. Parker. Real-Time Multiple Human Perception With Color-Depth Cameras on a Mobile Robot[J]. IEEE Transactions on Cybernetics. 43(5): 1429-1441(2013)

[7] J. H. Kim, D. H. Jang, K. J. Jang, S. Y. Lim. A study on object tracking for mobile robot using image and distance information[C]. 2016 16th International Conference on Control, Automation and Systems (ICCAS). IEEE, Gyeongju, South Korea. 1506-1508(2016)

[8] A. Basit, M. N. Dailey, P. Laksanacharoen, J. Moonrinta. Fast target redetection for CAMSHIFT using back-projection and histogram matching[C]. 2014 International Conference on Computer Vision Theory and Applications (VISAPP). IEEE, Lisbon, Portugal. 507-514(2014)

[9] W. C. Chang, C. H. Wu, H. C. Ling. A vision-based helper robotic system for the elderly in household environments[C]. 2014 CACS International Automatic Control Conference (CACS 2014). IEEE, Kaohsiung, Taiwan. 314-318(2014) 